\title{
Notas sobre os Oligochaeta da Amazônia
}

Gilberto Righi $\left({ }^{*}\right)$

\section{Resumo}

São estudadas três espécies de Oligochaeta da ilha Curarí, no rio Solimões. Novas ocorrências são registradas para Pristina minuta (Stephenson), Naididae, e Dichogaster andina evae Righi, Ayres \& Bittencourt, Octochaetidae, E descrita Hemienchytraeus (H) solimoensis, sp. n., Enchytraeidae.

Durante sua permanência no Instituto Nacional de Pesquisas da Amazônia, o Dr. Joachim Adis (Göttingen) fez uma grande coleção de animais, dos quais os Oligochaeta foram-me gentilmente enviados para estudo.

Os Oligochaeta estão representados nesta coleção por três espécies provenientes do solo de matas de várzeas na ilha Curari, rio Solimões, Amazonas $\left(03^{\circ} 21^{\prime} \mathrm{S}, 60^{\circ} 08^{\prime} \mathrm{W}\right)$. Estes animais encontram-se depositados no Instituto Nacional de Pesquisas da Amazônia (INPA), Manaus. Os estudos foram baseados em dissecções, em preparaçōes totais coradas pelo Paracarmin de Mayer ou Carmin borácico e em cortes seriados corados pelo método tríplice de Mallory (Pantin, 1964).

\section{NAIDIDAE}

Pristina minuta (Stephenson. 1914)

Naidium minutum Stephenson, 1914: 327, figs 3-5; 1923: 68, fig. 22; Marcus, 1943: 129, figs. 103 104A-C.

Pristina minuta, Sperber, 1948: 222; Stout, 1958: 291; Naidu, 1963: 206, figs. 29A-C; Brinkhurst, 1964: 217; Righi, 1973: 295, figs. 1-4.

MATERIAL - Brasil, Amazonas : ilha Curari, rio Solimões;: em solo de mata de várzea, 12 exemplares (INPAZ-96), J. Adis col. II-III/1976. DISTRIBUIÇÃo - Paquistão, Lahore (Stephenson, 1914: 437). India, Cuddaph (Naidu, 1963 : 206). Brasil, São Paulo : São Pedro (Marcus, 1943 : 129); Minas Gerais : serra do Cipó (Righi, 1973 : 295); Amazonas: ilha Curari.
Os animais da serra do Cipó e da ilha Curari vivem em solo úmido, periodicamente inundoda; os demais são límnicos.

\section{ENCHYTraeidae}

Hemienchytraeus (H.) solimoensis, sp. $n$. (Figs. 1-10)

MATERIAL - Brasil, Amazonas: ilha Curari, rio Solimões; em solo de mata de várzea, 11 exemplares (2 fragmentados) (INPAZ-97), J. Adis col. I-III/1976.

O comprimento dos animais varia de 5,0 a $8,5 \mathrm{~mm}$ e o diâmetro na região média do corpo de 0,2 a $0,3 \mathrm{~mm}$. O número desegmentos é de 30 a 36 . O prostômio é curto e cônico, com o poro cefálico subapical. Em cada segmento, há um anel de glândulas cutâneas coincidente com o anel setígero. Nos segmentos pré-clitelares pode haver mais um anel anterior e outro posterior por segmento. O clitelo é anelar em XII-1/2 e XIII e apresenta as células glandulares dispostas irregularmente.

A partir de II, há pares de tufos de cerdas por segmento, exceto em XII onde faltam. Há 2 certas por tufo, que se tornam sucessivamente maiores no sentido antero-posterior. Nos segmentos pré-clitelare o comprimento das cerdas varia de 30 a $37 \mu \mathrm{m}$ e nos pós-cli-telares de 50 a $56 \mu \mathrm{m}$. As cerdas são retas, sem nódulo e com ligeira curvatura ental (Fig. 1).

O primeiro par de nefrídios prende-se ao septo $6 / 7$; todos apresentam a massa celular intermediária bastante desenvolvida. A porção pré-septal é volumosa, constando do funil e algumas voltas do canal nefridial. A porção pós-septal é ovóide mais alongada nos nefrídios pós-clitelares. O canal excretor tem origem mediana ventral nos nefrídios pré-clitelares e póstero-ventral nos pós-clitelares (Figs.

$\left({ }^{\bullet}\right)$ - Departamento de Zoologia, Universidade de Sấo Paulo. 

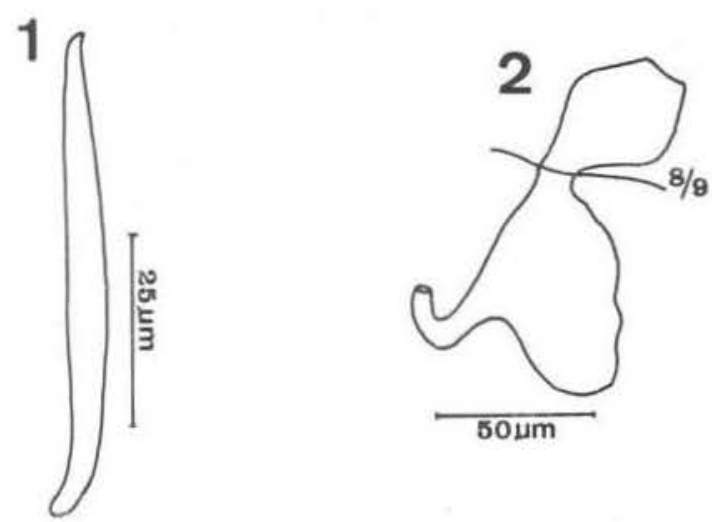

\section{5}

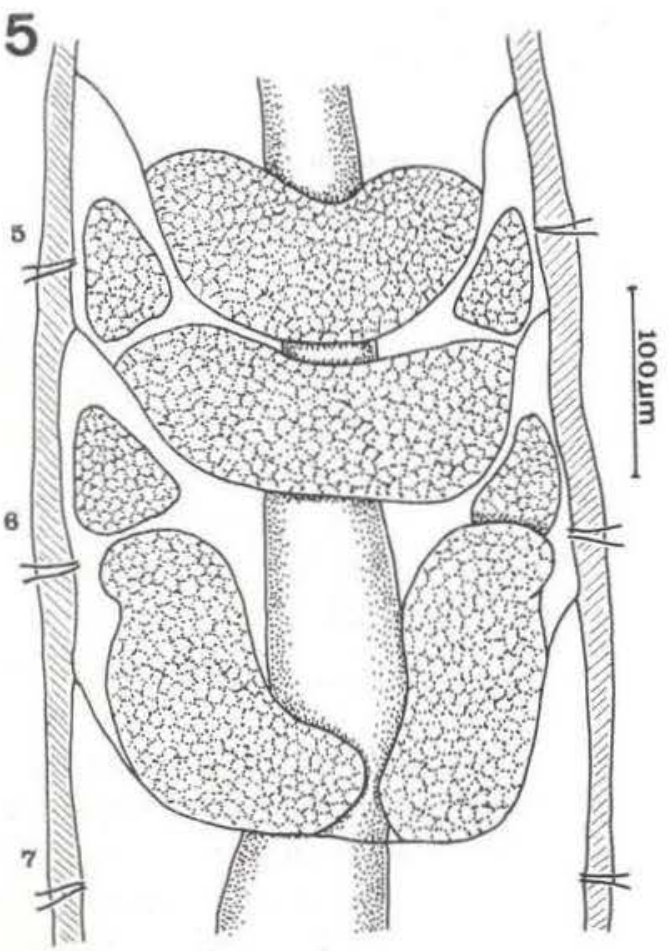

9

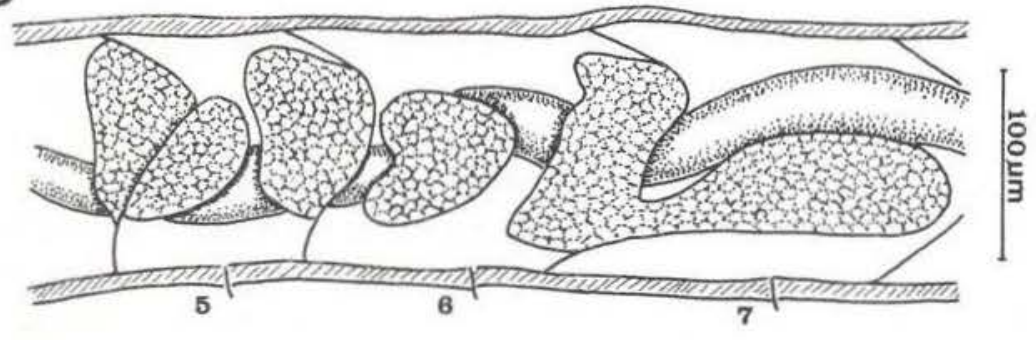

8
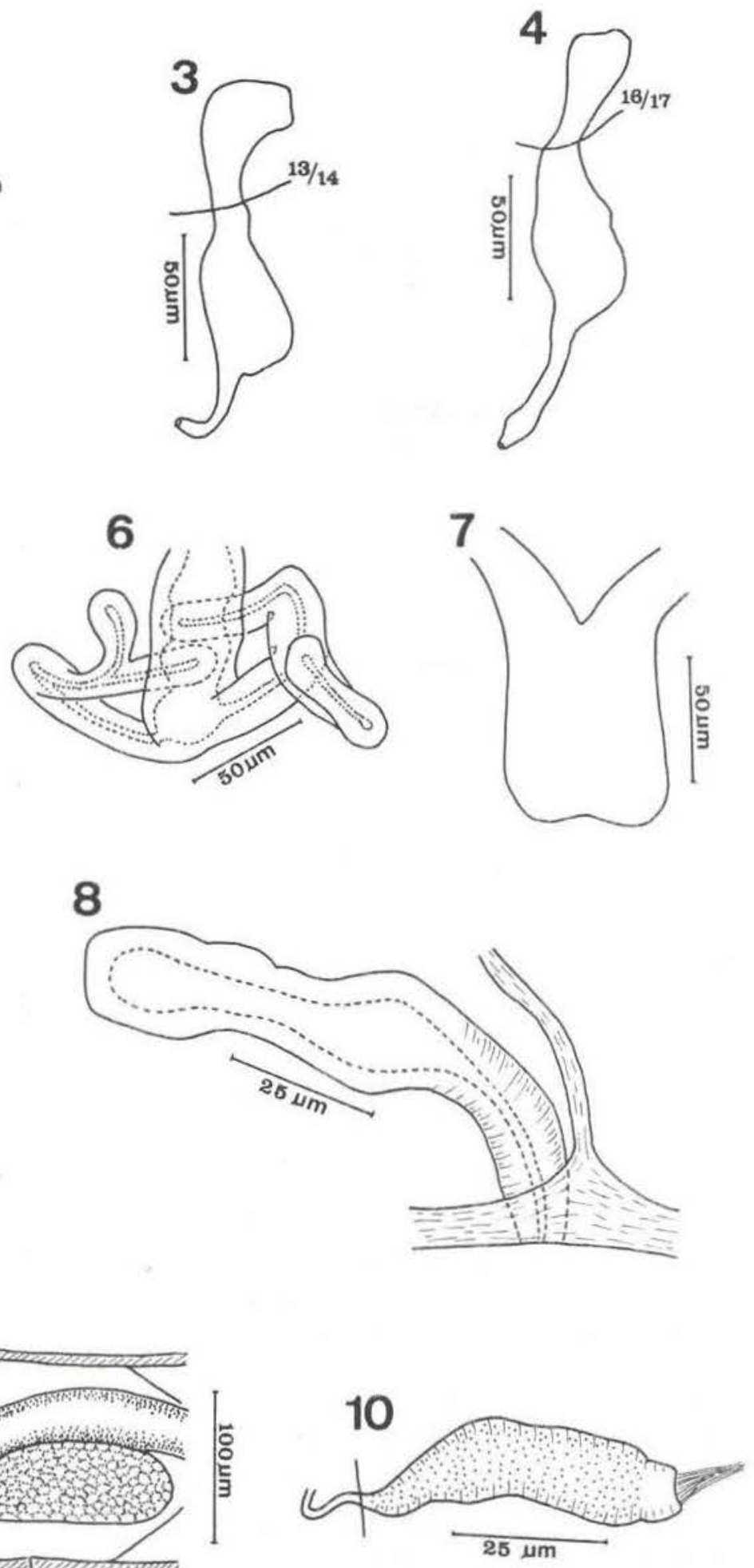

Hemienchytraeus (H.) solimoensis, sp. $\mathrm{n}$. -1 , cerda posterior; $2-4$, nefrídios; 5 , vista dorsal dos segmentos V-VII; 6, peptonefrídio; 7. gânglio cerebróide; 8, espermateca; 9, vista de perfil dos segmentos V-VII; 10, funil seminal. 
2-4). Os nefridióporos dispõem-se em série com as cerdas ventrais. A relação entre o comprimento das porçōes pré e pós-septal varia de $3: 2,8$, nos nefrídios pré-clitelares e de $2: 3$ ou $2,5: 3$ nos pós-clitelares. Celomócitos sã raros. Os poucos observados são discóides, com $9 \mu \mathrm{m}$ de diâmetro, nucleados e com o plasma dotado de numerosos grânulos refrigerantes. $\mathrm{O}$ gânglio cerebróide (Fig. 7) é pouco mais longo do que largo. A maior largura é próxima da margem posterior, que é côncava. As margens laterais convergem ligeiramente para a frente. O vaso dorsal inicia-se em 13/14.

A transição esôfago-intestino é gradual; não há divertículos. Células cloragógenas são reconheciveis a partir do segmento VII. O peptonefrídio é formado por uma porção basal cilíndrica, com larga cavidade irregular (Fig. 6). Distalmente divide-se em dois ramos primários de posição transversal, que se subdividem em dois ramos secundários. Estas ramificaçōes são pecrorridas por um canal delgado. Glândulas septais primárias bastante desenvolvidas prendem-se anteriormente nos septos $4 / 5$ e $6 / 7$ (Fig. 5). As simétricas dos dois primeiros pares fundem-se largamente sobre o esôfago; as do terceiro par são independentes. Pares de glândulas acessórias encontram-se em V e VI. Em um dos exemplares, as glândulas de $6 / 7$ prolongam-se ventralmente em VII (Fig. 9).

Um par de testículos maciços prende-se posteriormente em 10/11. Os funís masculinos têm a forma de cones alongados (Fig 10), 3 a 4 vezes mais longos do que a maior largura. Um colar cilíndrico é mal delimitado do restante do funil, porém distingue-se bem por suas células não apresentarem grânulos de inclusāo. Ao colar adere pequeno tufo de espermatozóides. Os canais deferentes são curtos, irregularmente espiralados e situam-se de cada lado, na região anterior e ventral de XII. Cada canal associa-se a um bulbo penial pequeno e arredondado, com cerca de $50 \mu \mathrm{m}$ de diâmetro. Poucos espermatozóides encontram-se na cavidade de XI. Vesícula seminal ausente.

Um par de ovários situa-se em XII. Um ovisaco volumoso prolonga-se dorsal e anteriormente até o septo $9 / 10 \mathrm{em} 8$ animais e pos- teriores até o septo $9 / 10$ em 8 animais e posteriormente até o segmento XIV em outros 3 . Ovários parciais (Teilovaria) e um ou dois ovos diferenciados encontram-se em seu interior $e$ em XII. Um par de espermatecas pequenas abre-se em $4 / 5$, em série com as cerdas dorsais e situa-se antero-lateralmente em V. Em cada espermateca (Fig. 8) o duto é musculoso, sem glândulas associadas e seu comprimento equivale a um terço do total da espermateca. A ampola dilata-se um pouco logo após o duto, a seguir diminui ligeiramente de calibre, e termina em outra pequena dilatação. Nenhum dos exemplares tem espermatozóides nas espermatecas.

\section{CONSIDERAÇÕES}

Pela organização dos peptonefrídios $\mathrm{He}$ mienchytraeus $(H$.$) solimoensis, aproxima-se$ de $H$. (H.) bifurcatus Nielsen \& Christensen, 1959, conhecida da Dinamarca. As duas espécies distinguem-se pelas espermatecas, origem do vaso dorsal e tamanho dos celomócitos. H. (H.) stephensoni (Cognetti, 1927) é uma espécie complexa, até agora, a única do subgênero conhecida da região Neotropical (Righi, 1974:130). H. (H.) solimoensis afasta-se de stephensoni pelas espermatecas e peptonefrídios. Năo está excluída a possibilidade de solimoensis ser apenas uma variedade de stephensoni, talvez uma linhagem partenogenética. Falam a favor desta última hipótese o tamanho reduzido das espermatecas, a pobreza de espermatozóides no segmento XI, sua ausência nas espermatecas e a posição aberrante do ovisaco na maioria dos animais.

\section{OCTOCHAETIDAE}

\section{Dichogaster andina evae Righi, Ayres \&} Bittencourt, 1977

D. andina evae Righi, Ayres \& Bittencourt, 1977, 6 figs.

MATERIAL - Brasil, Amazonas : ilha Curari, rio Solimões; em solo de mata de várzea, 5 exemplares clitelados (INPAZ-98), J. Adis col. II-III/1976. 


\section{CONSIDERAÇÕES}

Este material difere da forma típica da subespécie, conhecida apenas da regıāo do rio Preto de Eva, Amazonas, por faltarem papilas associadas aos poros prostáticos.

\section{SUMMARY}

Three species of Oligochaeta are studied from ilha Curari, river Solimōes, Amazonas, Brazil. New occurrences are recurded for the naidid Pristina minuta (Stephenson, 1914), and for the octochaetid Dichogaster andina evae Righi, Ayres \& Bittencourt, 1977. The enchytraeid Hemienchytraeus (H.) solimoensis, sp. n., is described.

\section{BIBLIOGRAFIA CITADA}

BRINKHURST, R.O.

1964 - Studies on the North American aquatic Oligochaeta. I. Naididae and Opistocystidae. Proc. Acad. Nat. Sc. Philadelphia, 116 (5):195-230.

COGNeTtI dE MARTHS, L.

1927 - Lumbricidi dei Carpazi. Boll. Mus. Zool. Genova (2)7(10):1-8.

MARCUS, E.

1943 - Sobre Naididae do Brasil. Bol. Fac. Fil. Ciênc. Let. Univ. S. Paulo, 25, Zoologia, 7:3-181, est. 1-33.

NAmU, K.V.

1963 - Studies on the freshwater Oligochaeta of South India. I. Aelosomatidae and Naididae, 5. J. Bombay Nat. Hist. Soc., 60 (1):201-227.
Nielsen, C.O. \& Christensen, B.

1959 - The Enchytraeidae. Critical revision and taxonomy of European species. Nat. Jut1., 8-9:1-160.

PANTIN, C.F.A.

1964 - Notes on microscopical technique for zoologists. : VIII $+76 \mathrm{pp}$. Cambridge University Press.

RIGHI, $\mathrm{G}$.

1973 - On Pristina minuta (Oligochaeta, Naididae) from Brazilian soil and its epizoic Rhabdostyla pristinis, sp. n. (Ciliata, Epistylidae). Zool, Anz., 19 (5/6):295-299.

1974 - Notas sobre os Oligochaeta Enchy. traeidae do Brasil, Papéis Avulsos S. Paulo, 28(7):127-144.

Righ, G., AYres, I. \& BITTENCOURT, E.C.R.

1977 - Oligochaeta (Annelida) do Instituto Nacional de Pesquisas da Amazônia Acta Amazonica (no prelo).

SPERBER, C.

1948 - A taxonomical study of the Naididae. Zool. Bidrag, 28:1-296, pls. 1-21.

STEPHENSON, J.

1914 - On a collection of Oligochaeta mainly from northern India. Rec. Ind. Mus., 10:321-365

1923 - The fauna of British India. Oligochae. ta. : XXIV +518 p., Taylor \& Francis, London.

Stout, $J$.

1958 - Aquatic Oligochaetes occurring in forest litter. II. Trans. R. Soc. New Zealand, 85(2):289-299

(Aceito para publicação em 20-08-78) 